\title{
Nuestra relación amor-odio con la Humanidad
}

\author{
Steve Fuller
}

xisten dos sentidos diferentes y no obstante superpuestos en
los que se podría realizar una 'sociología filosófica'. La obra Debating Humanity de Daniel Chernilo (2017a) lleva la impronta de ambas.

Por una parte, un 'sociólogo filosófico' puede entender su práctica sociológica como una expresión misma de ciertos temas y orientaciones filosóficas que se relacionan con la condición humana. Este tipo de reflexiones eran muy populares cuando estudié sociología en pregrado en la década de 1970. En ese entonces llevaba el nombre de 'sociología reflexiva'. Se orientaba a tratar los textos sociológicos como obras literarias, enfocadas principalmente en metáforas, giros irónicos y otro tipo de figuras retóricas, que servían para revelar y ocultar ciertos aspectos. Este acercamiento se enfocaba en el sociólogo como observador participante de los cambios que acontecían en su entorno. Se leían aquellos trabajos como fuentes de herramientas (quizás incluso de paradigmas) para construir una visión-demundo (Weltanschauung), la cual se formaría a partir de una combinación de la comprensión del pasado con las experiencias personales del presente, y que incluso se proyectarían hacia el futuro. Así, muy ligado al pensamiento

\footnotetext{
* Profesor "Auguste Compte" de Epistemología Social en el Departamento de Sociología de la Universidad de Warwick (Reino Unido). Asociado de la Academia Británica de Ciencias Sociales (UK Academy of Social Sciences), la Royal Society of Arts y la European Academy of Sciences and Arts. Email: S.W.Fuller@warwick.ac.uk

*^Este artículo consiste en la versión en español de la siguiente publicación: Fuller, Steve (2020) ‘Our love-hate relationship with humanity', Distinktion: Journal of Social Theory, 21(1), pp. 67-73. La traducción se ha realizado por Bernardo Villegas (Pontificia Universidad Católica del Ecuador). La publicación de este artículo se realiza con el amable permiso del autor y de Distinktion: Journal of Social Theory, a quienes agradecemos su contribución.
} 
de Karl Mannheim, toda la sociología sería 'siempre ya' una sociología del conocimiento. La 'sociología filosófica' de este estilo era popular a lo largo de todo el espectro ideológico, desde el neoconservadurismo de Robert Nisbet (1976) hasta el teórico crítico Richard Harvey Brown (1977). El grueso del libro de Chernilo tiene un poco de este estilo de 'sociología filosófica’, aunque su análisis es más escolástico y menos literario.

Por otra parte, la 'sociología filosófica' podría ser otra manera de hablar de la 'antropología filosófica' que intenta comprender la totalidad de los fenómenos humanos como expresión de algún aspecto filosófico sobre la naturaleza de la humanidad. En breve, sobre la pregunta ¿qué significa ser ‘humano'? El primer autor que reclamó el título de 'antropólogo filosófico' sería uno de los fundadores de la sociología del conocimiento, Max Scheler, a quien Chernilo hace referencia en su introducción y conclusión. Estos son sin embargo realmente los únicos lugares donde el autor chileno persigue el sentido más ambicioso de la 'sociología filosófica', un hecho que podría decepcionar a algunos lectores, debido a que Chernilo evidentemente tiene clara la significancia de 'debatir la humanidad' en este momento en el que nos abrimos a futuros de 'trans-' y 'post-' humanos. En este caso, su libro se puede leer generosamente como un ejercicio 'propedéutico' (metafóricamente, como un ejercicio de calentamiento de motores) de un proyecto más largo, y se podría juzgar mejor por los hallazgos de los teóricos allí considerados a la luz de lo que he denominado como 'Humanidad 2.0' (Fuller, 2011). Dudo que muchos de los pensadores a los que Chernilo brinda capítulos enteros sean incluidos o referenciados en un libro de teoría social llamado 'Debatiendo la Humanidad' que podría aparecer en, digamos, un cuarto o medio de siglo. De todos modos, el libro de Chernilo seguramente será de gran interés para futuros historiadores que deseen comprender las 
dificultades con las que lidian los académicos rigurosos de hoy en día para trazar el futuro de nuestra especie.

En este marco, es valioso recordar el ambicioso estándar que Scheler fijó. A pesar de que murió en 1928, antes de finalizar su opus magnum sobre la 'antropología filosófica', Scheler dejó una pequeña pero sustancial cantidad de trabajo que provee un sentido razonablemente claro de los asuntos relevantes (Scheler, 2009). El libro apareció en 1961, por primera vez en inglés, con el desafortunado título de El lugar del Hombre en la Naturaleza (Man's Place in Nature); si bien una traducción más reciente y literal transmite el sentido original de Scheler: El lugar Humano en el Cosmos (The Human Place in the Cosmos). Para Scheler, la necesidad de una antropología filosófica surge porque lo 'humano' puede referirse o bien a especies animales o a seres que han trascendido totalmente su condición animal. En estos términos, ¿es el Homo Sapiens una especie más entre otras (e pluribus unum) o una especie radicalmente diferente (sui generis)? Al final del siglo XIX, Darwin sostuvo la primera postura, Durkheim la posterior. Así comenzó el cordon sanitaire entre la biología y la sociología, la cual se mantuvo en buena parte del siglo XX, especialmente después de las políticas eugenésicas que fueron las causantes del Holocausto (Fuller, 2006). De todos modos, la antropología filosófica de Scheler estaba orientada a reabrir la pregunta que surgió en el ocaso de la Primera Guerra Mundial, en el mismo modo en que se ha replanteado en esta época, casi un siglo después.

Pero la situación actual es complicada, no solamente por la inestabilidad normativa e incluso descriptiva del Homo Sapiens, sino también por el sitio en el que lo 'humano' se despliega y performa. Sucede que, no sólo Scheler y los seguidores en la antropología filosófica -incluyendo a Arnold Gehlen 
y Helmuth Plessner- comprendieron el carácter que tiene el Homo sapiens como especie en términos 'tipológicos', sino que también lo han hecho los teóricos a quienes Chernilo dedica capítulos individuales. Esto podría explicarse debido a que los términos 'tipológicos' son más informativos que los términos biológicos, al cual los sociólogos calificarían de 'esencialistas'. Un pensador tipológico explica las variaciones individuales como elementos organizados alrededor de un implícito estado normal. Alguien que piensa de esta manera tiende a buscar individuos que ‘ejemplifican’ el tipo en cuestión, quienes están más cerca de la norma asumida. Los biólogos llamarían a tal individuo como un 'espécimen'. El fundamento cognitivo de este último modo de pensar yace en la perspectiva Aristotélica que busca unidad en diversidad, a saber, intuir la esencia de cada uno de los individuos relevantes despliega, incluso a pesar de las variaciones graduales que se encuentren. Este autor llama a este proceso epagoge, lo que después de Charles Sanders Pierce se suele traducir provocativamente como 'abducción'. En cualquier caso, el pensamiento tipológico favorece al sensible investigador cualitativo sobre el escrupuloso investigador cuantitativo. Cuando los dos se combinan -como en El Suicidio de Durkheim- el resultado tiende a traer consigo fuertes implicaciones normativas asociadas con las desviaciones positivas y negativas de una supuesta media. Este es el mundo de la 'curva de la distribución normal', en el cual frases como 'problemas sociales' y 'progreso social' tienen sentido.

De todos modos, tal como el famoso taxonomista de Harvard entrenado en Alemania Ernst Mayr (1959) argumentó famosamente en el centenario retrospectivo de El origen de las especies, Darwin efectivamente deconstruyó la concepción tipológica de las especies como una simple manera conveniente de captar líneas de aptitud y ajuste reproductivo de individuos independientemente su origen. Así, Darwin marcó el 
advenimiento de lo que Mayr llamó el 'pensamiento poblacional', el cual empieza a brindar a las estadísticas un lugar especial en el ámbito cognitivo sobre cómo se definen y evalúan los patrones de vida. Esto, a su vez, ha brindado mayor alcance a la imaginación humana para promover y/o inhibir el destino de los individuos. Si bien la eugenesia era la vanguardia histórica de este enfoque, algunas versiones más blandas continúan promoviendo la 'movilidad social' en las políticas de los Estados de Bienestar, las cuales brindan conceptualmente una aguda percepción acerca de la fluidez de la 'sociedad'. En este sentido, si la política gubernamental consiste en permitir que los individuos puedan triunfar en cualquier ámbito donde lo deseen, entonces, la identidad del todo estará compuesta por la suma de estas partes, y la 'sociedad', a su vez, seguirá siendo una entidad indeterminada. Muchos acogen este desarrollo como 'el precio de la libertad'. Sin embargo, quizás también ayuda a explicar los motivos por los que, en los últimos treinta años, la 'sociología' en el estricto sentido durkheimiano ha presentado su debacle. Como el concepto de 'especies' después de Darwin, la 'sociedad' en sí misma se ve, cada vez más, como una construcción social. En este contexto, a menos que la sociología se vuelva 'global' o 'cósmica', en un sentido relevante, es posible que pronto deje de existir como una disciplina coherente. Entre muchas personas, Lester Ward, el primer presidente de la American Sociological Association (ASA), ha brindado ciertas luces sobre este asunto.

Lo que acabo de describir como la transición del pensamiento tipológico hacia el pensamiento poblacional es un giro de 'segundo orden' en nuestra comprensión del 'humano', pues se preocupa del marco metafísico dentro del cual se ha conceptualizado al humano. No obstante, la perspectiva original de Scheler se complejiza por un giro de 'primer orden', que desestabilizó de distintos modos la esencia de la herencia evolutiva de la humanidad. 
Usualmente, los antropólogos filosóficos han aceptado el amplio bosquejo de la evolución como la prehistoria de la humanidad, a pesar de que no necesariamente se encuentran en total acuerdo con el Darwinismo. Para ellos ha significado aceptar que, incluso si el Homo Sapiens no fuese la especie más 'avanzada', queda claro que es un ser excepcional, y una tarea importante de la antropología filosófica es comprender esa excepcionalidad como un hecho natural y una prescripción normativa sobre nuestro futuro como especie. Hay dos movimientos contemporáneos que desafían profundamente esta comprensión del humano. El posthumanismo, por un lado, el cual observaría a los humanos como seres finitos dentro de un ecosistema donde sus características 'excepcionales' han producido el oscuro origen del 'Antropoceno' (Chernilo 2017b). Por otro lado, el transhumanismo que ubica al excepcionalismo humano en nuestra capacidad para proyectarnos indefinidamente, donde incluso algunas de sus versiones apuntan a la posibilidad de escapar no solamente de nuestros cuerpos simiescos sino del planeta Tierra. Mientras los posthumanistas intentan descentrar al humano como el centro del universo, los transhumanistas buscan reforzar su valor. De esta manera, posthumansmo y transhumanismo retoman en un nuevo registro la pregunta de Scheler acerca de si el Homo Sapiens es una especie más entre otras (e pluribus unum) o una especie radicalmente única (sui generis). Chernilo acertadamente asocia el posthumanismo con Donna Haraway y Bruno Latour, mientras que yo cada vez me he identificado más con el transhumanismo (Fuller \& Lipinska 2014).

Es valioso mencionar que la sociología ha sido muy lenta en tomar con seriedad esta emergente distinción. A pesar de que el despegue humano hacia el espacio exterior en la década de 1960 produjo campos como la 'exosociología' y la 'sociología cósmica', que concebían a todo el universo como una sociedad, estas perspectivas han operado de modo ortogonal 
en relación a lo que distingue a los post- y transhumanistas (Dickens and Ormrod 2007, Schetsche and Anton 2019). En consonancia con posthumanistas, imaginan a la forma humana como una entidad emparejada al Homo Sapiens, al tiempo que - al igual que transhumanistas - imaginan que el Homo Sapiens podría encontrar todo tipo de experiencias totalmente ajenas a su especie. Los posthumanistas aceptan la primera premisa, pero no la segunda; en cambio, los transhumanistas aceptan la segunda premisa, pero no la primera. Lo que se encuentra en medio de ambas posiciones es el estatus del 'cyborg', entendido como un ser que nació como Homo Sapiens, pero que a lo largo de estos 40.000 años (la fecha aceptada actualmente en la que el cerebro humano habría adquirido sus habilidades actuales) ha mejorado de múltiples maneras, con lo cual difiere significativamente de su origen biológico. Así, Chernilo comprende en medida correcta la distinción entre el post-/trans- humanismo en su introducción de Debating Humanity, cuando evita etiquetar como 'pos-humanista' a la tesis de la 'mente extendida' de Andy Clark, simplemente porque permite que lo 'humano' designe a un agente distribuido físicamente. En cambio, la pregunta crucial es si el humano biológico -más específicamente, el cerebro- continúa siendo responsable vis-à-vis las múltiples fuentes externas de la memoria o de los motores que la potencian (las cuales Clark visualiza como elementos constitutivos de la futura existencia cyborg de la humanidad).

Sobre este asunto coincido; aunque quizás, a diferencia de Chernilo, agregaría que, en la medida en que la 'naturaleza humana' es un concepto coherente, los humanos somos por naturaleza 'morfológicamente libres', a pesar del origen biológico o nuestra corporización actual (Fuller, 2019). Efectivamente, se puede pensar al humano como una entidad corporativa en el sentido reconocido por la ley Romana a finales de la Edad Media. Por ejemplo, una empresa puede ser una ‘corporación', incluso a pesar de que 
sus constituyentes físicos están ampliamente distribuidos, reorganizados y reemplazados a lo largo del tiempo - pues, sin embargo, mantiene cierta continuidad en su propósito o misión. Lo mismo aplica para individuos humanos, y esto ha sido ampliamente reconocido por la ley. Entonces, la identidad de una persona está ligada a sus activos y pasivos, 'sus herencias', en un marco en el que las futuras generaciones están encomendadas a emplear. John Locke psicologizó esta tendencia rápidamente en los inicios de la era moderna, cuando definió la identidad personal en términos de continuidad de la memoria, en cuyo caso resulta importante la decisión de una persona acerca de cómo debe 'continuar' después de su muerte. Hay una expectativa social de continuidad, sea en la forma de un 'testamento y último deseo', una nota de suicidio o, actualmente, un legado digital en redes sociales. Por su parte, Scheler comprendió esta expectativa como una expresión del sentido de auto-trascendencia de la humanidad, la cual respondería, en último término, a la necesidad de enmarcar eventos discretos como partes de una totalidad infinitamente más amplia, cuya 'personalidad' está moldeada a lo largo del tiempo. Este fue el sentido metafísico sobre el (así llamado) "sentido de la vida", que Scheler parecía identificar especialmente con el retorno hacia Dios.

Los intereses y ecos teológicos encontrados en el trabajo de Scheler no son atípicos, pero necesarios en cualquier discusión profunda sobre el 'humano', cuya importancia normativa y descriptiva data de antes de la acuñación de la palabra 'Homo Sapiens', realizada por Linnaeus, a medidos del siglo XVIII. A pesar de que Scheler fue un pensador tipológico del humano, la distinción entre pensamiento poblacional y tipológico también estuvo rudimentariamente presente en los intentos medievales por definir al humano como un ser dirigido por lo animal y lo divino. Sobre esta discusión, cabe resaltar la distinción lógica entre el sentido extensional e intencional 
del término 'humano'. Por ejemplo, la 'humanidad' bien se puede referir a individuos específicos a quienes clasificamos dentro de un mismo género como 'humanos' (extensional), o bien a ciertas propiedades subyacentes que esos individuos comparten y que los hacen humanos (intencional). A finales de la Edad Media, esta distinción adquiere importancia metafísica como un debate escolástico sobre el lenguaje humano entre el nominalismo y el realismo, en relación al lenguaje humano, entendido como el medio privilegiado, aunque con imperfecciones, mediante el que los humanos alcanzan una comprensión de la creación divina. Los nominalistas enfatizaban el sentido bíblico de la humanidad, que básicamente implica que nuestro lenguaje está sujeto a error, por lo cual siempre habrá una potencial necesidad de revisarlo. Los realistas, en contraste, sostenían una actitud más optimista sobre el lenguaje, pues creían que normalmente capturaba el mundo tal y como es, lo cual ayudaba a explicar la supervivencia y el desarrollo humano. Para resumir una historia larga y complicada, los nominalistas son los antecesores de la 'revolución científica' Occidental, mientras que los realistas defienden el status quo Católico Romano.

En el periodo moderno, las preguntas medievales se realizaron más puntualmente: ¿se trata de un accidente histórico que se ha vuelto natural el hecho de clasificar a ciertos como 'humanos'? Si es así, entonces se debería preguntar en qué momento sucedió que tales individuos deberían ser catalogados como tales, o incluso, si es útil la categoría 'humanos'. Hay pocas dudas de que esta peligrosa línea de argumentación pertenece al legado intelectual del nominalismo. En realidad, se encuentra en la base de las preocupaciones que surgieron originalmente por Horkheimer y Adorno (1972) sobre la ‘deshumanización’ que se vivía a través del triunfo de la 'racionalidad instrumental', cuyo peso cayó encima del primer gran nominalista moderno e inventor del método científico, Francis Bacon. La 
crítica adquirió un contrapeso normativo adicional a inicios de la Segunda Guerra Mundial, el cual Chernilo elabora y amplifica, especialmente en sus capítulos de Hans Jonas y Hannah Arendt. Me permitiré dos observaciones. Primero, la crítica al nominalismo, cuya cara positiva es una especie de 'humanismo sin ilusiones', considera a las atrocidades tecnocientíficas del siglo XX no sólo como aberraciones históricas, sino también una señal del mal que los humanos son capaces de producir si se resisten a reconocer su propia finitud. Segundo, el imperativo moral que se deriva de esta crítica, según la cual mejor conocimiento humano conlleva una mayor responsabilidad, se interpreta en términos expresamente preventivos. En pocas palabras, debemos hacer la menor cantidad de daño, e idealmente dejar la Tierra en no peores condiciones de las que encontramos.

Sin embargo, la rama nihilista del nominalismo que derivó en el Holocausto, que acecha a esta crítica, no ha sido la tendencia dominante en toda la historia. De hecho, la explosión de la bomba atómica sobre Hiroshima vino de la mano con avances en la tecnología nuclear sin derivar en una Tercera Guerra Mundial. Por el contrario, la tendencia dominante ha estado más cercana a la opinión de Leibniz, en el sentido de que la tarea de la humanidad es convertir sus limitaciones naturales en virtudes artificiales dentro de un mundo que, en último término, se encuentra observado por una deidad justa y benevolente. Podríamos ser seres caídos, en el sentido bíblico, pero nunca estaremos condenados ni siquiera quizás 'arrojados' (en el sentido heideggeriano de la palabra geworfen). Así, incluso si algo tan básico como nuestro lenguaje nos conduce a confundirnos, nosotros, no obstante, seguimos siendo libres para -e incluso se podría decir que estamos obligados a-figurar formas alternativas de comprender el mundo dentro de una mentalidad experimental. La pregunta normativa que sigue abierta es si 
este ejercicio apunta a que nos ajustemos al mundo tal como lo encontramos -lo que sugiere cierta pasividad política-, o cambiarlo para permitirnos formas de autoexpresión en búsqueda de recuperar nuestras habilidades divinas pre-celestiales. Lo último implica la política proactiva que ha tenido a la ciencia como su punta de lanza - para el bien o para el mal- a lo largo de los últimos cuatro siglos. En este contexto, es sorprendente que el biólogo evolutivo, Julian Huxley, que acuño el término 'transhumanismo' en la década de 1950, elabora exactamente el mismo argumento que Jonas acerca de la 'responsabilidad hacia el mundo', con la diferencia de que Huxley concluye que deberíamos acelerar el camino de la evolución mediante la eugenesia.

En cambio, para los realistas, la palabra 'humano' se refiere a un todo que es más que la suma de sus partes, lo cual trae de vuelta la raíz latina de 'concepto' en tanto 'agarrar en conjunto'. La idea es que la 'humanidad' como concepto se revela únicamente cuando todos los individuos se encuentran dentro de una unión implícita, de una unidad. Esto ayuda a explicar el marco de referencia original de la Edad Media sobre la ley natural, donde el valor de todos los seres del 'universo' estaba determinado según el rol que tenían para mantener al todo. El legado moderno de esta idea fue uno de los temas de los anteriores libros de Chernilo (Chernilo 2013). Es innegable que el universo medieval estaba organizado jerárquicamente, lo cual desde un punto de vista moderno parecería estar basado en un principio de desigualdad genética. De todos modos, como lo observó primero Hegel y luego Marx, la dialéctica del amo y el esclavo revela que incluso el universo medieval suponía la igualdad como una propiedad de 'segundo orden' de la humanidad, pues, el mantenimiento de esta jerarquía requiere el respeto y reconocimiento mиtuo entre todos los individuos, sin importar su posición asimétrica en la sociedad. El llamado de Marx a la unión de todos los trabajadores para que 
dejen a un lado su labor, en nombre de la 'conciencia de clase', apuntaba a demostrar que la dependencia que tienen los capitalistas de ellos (era, en el fondo, la misma que su dependencia de los capitalistas.

Nuevamente, la intuición religiosa original resulta clarificadora. No es suficiente que cada persona entienda su lugar dentro del plan de Dios. También deben comprender la necesidad del rol de los otros para que el plan funcione. Dicho de otro modo, se deben reconocer a sí mismos en otros. (Se escucha el eco pragmatista de la sociología norteamericana de Geoge Herbert Mead y Charles Horton Cooley - y también la raíz Aristotélica epagoge, al observar la unidad en lo individual). Este sentido formal de igualdad se ha secularizado en la era moderna como una libertad generalizada para formar asociaciones, incluso compañías, partidos políticos y estados-nación. En todos estos casos, los individuos acuerdan voluntariamente identificarse entre sí como iguales, y a sí mismos como elementos dentro de un todo más amplio. El contrato social es el mito fundante de esta perspectiva, en la cual Dios no es parte de estas negociaciones -a pesar de que una deidad ha sido creada durante este proceso, el Dios-Estado a la Leviathan de Hobbes. En relación a esto, se podría argumentar que Marx intentó mostrar al materialismo histórico como una alternativa a la pérdida metafísica de una deidad providencial, puesto que, a su juicio, la versión del contrato social sería insuficiente para unificar a la humanidad. Actualmente, el estándar canónico de la humanidad ha sido establecido por la Declaración Universal de Derechos Humanos de las Naciones Unidas, la cual ha sido prevista por el filósofo moderno católico, Jacques Maritain, quien conjugó hábilmente la comprensión de humanidad medieval-cooperativista, liberal-contractualista y marxista-emancipatoria, y la transformó en la piedra angular del Derecho Internacional que ha durado por setenta años (Moyn 2011). 
De este modo, la emergencia del posthumanismo y del transhumanismo reaviva en otros enfoques el debate entre el nominalismo y el realismo sobre el significado del 'humano'. Cada movimiento contiene elementos de alguno de los dos lados del debate. En su siguiente libro Chernilo podría orientar su mirada analítica de manera más directa a este tipo de debates de avanzada, quizás adaptando la frase 'sociología liminal' de Arpad Szakolczai (2000), que aparece en un libro comparable en muchos sentidos a Debating Humanity. A modo de conclusión, quisiera traer a colación a una manera moderna y provocativa de reconciliar la comprensión nominalista y realista del humano, que claramente tiene potencial de adquirir más sentido en el futuro. Esto también trae de vuelta a una de las caracterizaciones distintivas de una 'vida humana con sentido' de Scheler. Se puede considerar el aforismo: 'Amo la humanidad. Son las personas a quienes odio'. Si bien esta frase ha sido atribuida a varios personajes (entre ellos, Robespierre, Einstein, el poeta norteamericano Eda St. Vicen Millay y al caricaturista norteamericano Charles M. Schulz (famoso por Peanuts)), creo de todos modos, que la versión más interesante es quizás la que aparece en Los Hermanos Karamazov de Dostoievsky. Allí se dice que mientras más se ama a la humanidad, una mayor cantidad de humanos individuales parecerían deficientes. En ambos casos, en el posthumanismo y en el transhumanismo, este sentimiento se encuentra muy presente, aunque en direcciones opuestas. Para uno los 'humanos' son esencialmente animales (pero los individuos humanos son lentos al reconocer este hecho), mientras que, para los otros, el 'humano' es un ser que trasciende lo animal (pero, de igual modo, los individuos humanos son lentos para reconocerlo). Incluso el mismo Scheler se refirió a este asunto en términos criptocristianos. Señaló que el odio a los límites en nuestro ser constituyen los medios para superar la tentación del amor propio y, de esa manera, nos abren hacia el amor incondicional 
que conduce hacia el conocimiento de Dios. Desde una perspectiva secular, podría interpretarse acertadamente a Scheler como trazando la dirección del viaje metafísico hacia el cual es posible que vayan a llegar los futuros debates sobre la humanidad.

\section{Referencias}

Brown, R.H. (1977). A Poetic for Sociology. Cambridge UK: Cambridge University Press.

Chernilo, D. (2013). The Natural Law Foundations of Social Theory. Cambridge UK: Cambridge University Press.

Chernilo, D. (2017a). Debating Humanity: Towards a Philosophical Sociology. Cambridge UK: Cambridge University Press.

Chernilo, D. (2017b). 'The question of the human in the Anthropocene debate', European Journal of Social Theory 20 (1): 44-60.

Dickens, P. and Ormrod, J. (2007). Cosmic Society. London: Routledge.

Fuller, S. (2006). The New Sociological Imagination. London: Sage.

Fuller, S. (2011). Humanity 2.0: What It Means to Be Human Past, Present and Future. London: Palgrave.

Fuller, S. (2019). Nietzschean Meditations: Untimely Thoughts at the Dawn of the Transhuman Era. Basel SZ: Schwabe Verlag.

Fuller, S. and Lipinska, V. (2014). The Proactionary Imperative: A Foundation for Transhumanism. London: Palgrave.

Horkheimer, M. and Adorno, T. (1972). The Dialectic of Enlightenment. (Orig. 1944). London: Continuum.

Mayr, E. (1959). “Typological versus Population Thinking.” In B. J. Meggers (ed.), Evolution and Anthropology: A Centennial Appraisal, 409-12. Washington DC: Anthropological Society of Washington. 